\title{
Wettbewerbliche Banken fördern das Wachstum
}

\author{
Verena Maria Konzett
}

\section{Relevanz}

Was nützen die besten Ideen, wenn Investitionen und neue Jobs mangels Zugang zu Bankkrediten nicht zustande kommen? Wachstum und Innovation brauchen einen leistungsfähigen Finanzsektor. Wettbewerb regt auch die Banken zu Höchstleistungen an. Indem sie mehr Informationen über ihre Kunden sammeln und ihre Prozesse bei der Auswahl und anschliessenden Überwachung optimieren, gelingt es ihnen besser, die besonders vielversprechenden Unternehmen $\mathrm{zu}$ identifizieren. Dadurch, dass die Banken die Kreditvergabe vor allem auf die innovativen und profitablen Unternehmen mit hohem Wachstumspotential lenken, fördern sie die Produktivitätssteigerungen und das Wachstum der Realwirtschaft.

\section{Quelle}

Bai, J., D. Carvalho, und G. M. Phillips (2018), The Impact of Bank Credit on Labor Reallocation and Aggregate Industry Productivity, Journal of Finance 63(6), 2787-2836.

Ein gut funktionierender Finanzsektor stärkt das Wachstum. Leistungsfähige Banken und liquide Kapitalmärkte können die wirtschaftliche Entwicklung über viele Wege anstossen. Weniger klar ist, welche speziellen Mechanismen wichtig sind und wie genau der Finanzsektor das Wirtschaftswachstum beeinflusst. John

V. M. Konzett $(\bowtie)$

Universität St.Gallen, St.Gallen, Schweiz

E-Mail: verena.konzett@student.unisg.ch 
Bai, Daniel Carvalho und Gordon Phillips untersuchen empirisch die Rolle von Banken für Produktivität und Wachstum der Realwirtschaft. Können Banken mit gezielter Vergabe von Krediten die Ressourcen zu den produktivsten Unternehmen lenken? Wie stark ist ihr Beitrag zu Produktivität und Wachstum in der Realwirtschaft? Wie können die regulatorischen Rahmenbedingungen die Qualität der Kreditvergabe verbessern?

Die Wissenschaftler untersuchen die Kreditvergabe in den USA während der 1980er Jahre. Der Bankensektor war bis zum Ende der 1970er Jahre von starken staatlichen Eingriffen geprägt, welche die Entscheidungen der Banken behinderten und den Wettbewerb auf den lokalen Kreditmärkten einschränkten. So war die Geschäftstätigkeit einer Bank typischerweise auf einen bestimmten Bundesstaat beschränkt. Der erschwerte Marktzutritt begünstigte monopolähnliche Strukturen auf den lokalen Bankenmärkten. Dabei waren zwei Einschränkungen besonders wichtig. Erstens war die Eröffnung neuer Filialen innerhalb eines Bundesstaates beschränkt, wie der Intra-Deregulierungsindex in Abb. 1 illustriert. Letzterer misst die Strenge der innerstaatlichen Regulierung auf einer Skala zwischen 0 (starke Eingriffe) und 1 (schwache Eingriffe). Zweitens

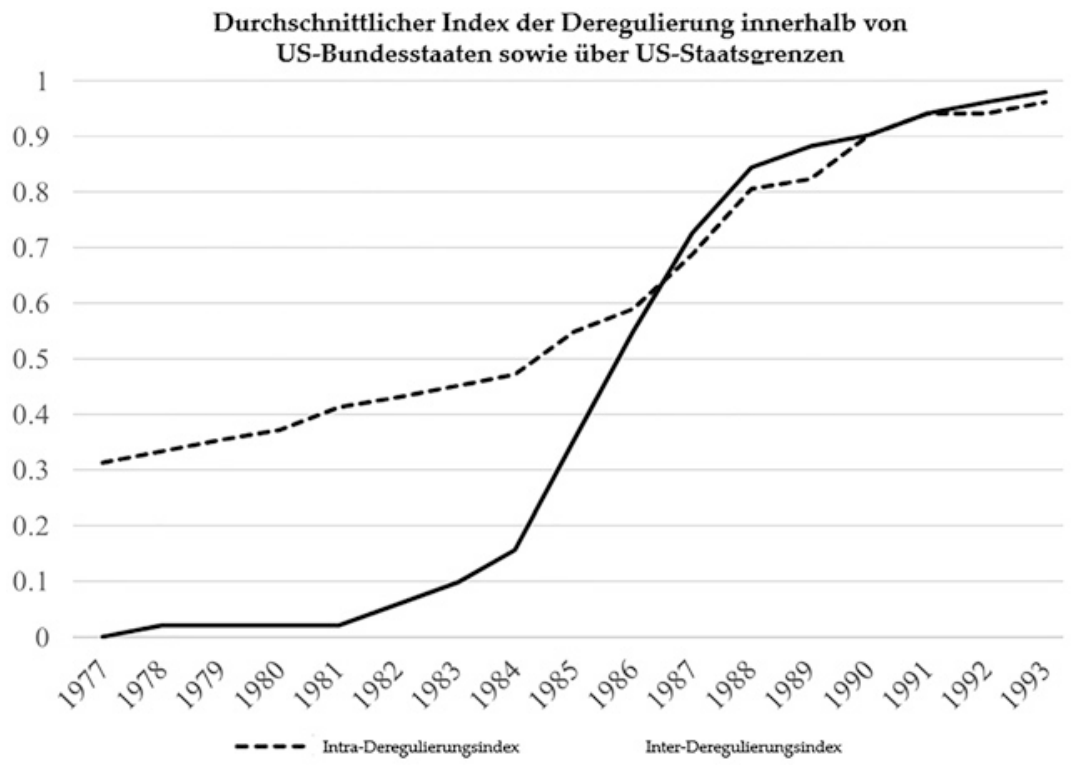

Abb. 1 Deregulierung der Bankenmärkte in US-Bundesstaaten. (Quelle: Bai et al. 2018) 
war es Banken und Holdinggesellschaften meist untersagt, eine andere Bank mit Sitz ausserhalb des Bundesstaates zu erwerben. Solche Restriktionen fasst der vergleichbare Inter-Deregulierungsindex in Abb. 1 zusammen. Zwischen 1977 und 1993 deregulierten die meisten Bundesstaaten ihre lokalen Bankenmärkte und bauten zahlreiche Beschränkungen und Marktzutrittshürden ab. So wurden etwa Übernahmen von Banken in anderen Bundesstaaten erlaubt.

Die Deregulierung der Bankenmärkte in den USA verschärfte den Wettbewerb zwischen den Banken und beeinflusste deren Kreditvergabe stark. Der Wettbewerb zwang die Banken, ihre Prozesse bei Auswahl und Überwachung (,Monitoring') von Kreditnehmern zu verbessern. Indem sie mehr Informationen über ihre Kunden sammelten, konnten sie produktive und vielversprechende Unternehmen genauer identifizieren und besser überwachen. Dies senkt nicht nur die Kreditausfälle, sondern trägt auch dazu bei, dass die Unternehmen mit den erhaltenen Krediten höhere Erträge erwirtschaften und stärker wachsen. Speziell führten die Veränderungen dazu, dass die Banken einen grösseren Anteil ihrer Kredite gezielter an besonders produktive Unternehmen vergeben konnten. Bankkredite sind für kleine und junge Unternehmen von besonderer Bedeutung. Sie sind oft geographisch an einen Bundesstaat gebunden und profitieren daher vom Marktzutritt neuer Banken. Zudem spielen Unterschiede im Auswahl- und Überwachungsprozess für junge Unternehmen eine grössere Rolle, da sie meist noch über keine Reputation als zuverlässige Schuldner verfügen.

Die Wissenschaftler verwenden einen Datensatz mit Informationen zu kleinen Industrieunternehmen in den USA (1977-1993). Bei Ihnen stellen Bankkredite eine besonders wichtige Finanzierungsquelle dar. Sie repräsentieren zwischen 50 und $60 \%$ ihrer Verbindlichkeiten. Im Durchschnitt finanzieren die Banken rund $30 \%$ des Firmenvermögens. Bei jungen Unternehmen, welche höchstens zehn Jahre alt sind, beträgt dieser Anteil sogar $40 \%$.

Nach Deregulierung der Bankenmärkte nimmt der Anteil der Bankkredite an der Gesamtverschuldung bei jungen Unternehmen acht Mal so stark zu wie bei älteren Unternehmen.

Die Forscher argumentieren, dass produktive Unternehmen überproportional von den Veränderungen des Kreditangebots profitieren. Ihr Potenzial wird von den Banken besser erkannt. Sie erhalten daher leichter Kredite, sodass sie schneller wachsen können. Die empirischen Ergebnisse zeigen zunächst, dass sich solche Unternehmen aufgrund der Deregulierung stärker bei Banken finanzieren können. Dabei nimmt der Anteil der Bankkredite an der Gesamtverschuldung bei hochproduktiven Unternehmen stärker zu. Dies deutet darauf hin, dass Banken die 
Kredite gezielter an solche Firmen vergeben. Zudem ist der Effekt bei jungen Unternehmen rund acht Mal stärker ausgeprägt als bei Älteren.

Die empirischen Ergebnisse zeigen weiter, dass Deregulierung und stärkerer Wettbewerb auf lokalen Bankenmärkten vor allem das Wachstum besonders produktiver Unternehmen steigert. Solche Unternehmen vergrössern ihre Belegschaft und erhöhen ihren Kapitaleinsatz in Folge des intensiveren Wettbewerbs im Bankensektor stärker als weniger produktive Firmen. Allerdings ist dieser Effekt nur bei jungen Unternehmen statistisch signifikant. In dieser Gruppe erhöht sich beispielsweise das Beschäftigungswachstum, welches durchschnittlich 5,5\% beträgt, bei hochproduktiven Firmen um 3 bis 4,5 Prozentpunkte.

Die Deregulierung der Bankenmärkte erhöht das Beschäftigungswachstum von jungen, produktiven Unternehmen um 3 bis 4.5 Prozentpunkte mehr als jenes von weniger produktiven Firmen.

Zuletzt quantifizieren die Forscher die Produktivitätsgewinne innerhalb einer Branche. Produktiver Unternehmen wachsen Dank gezielter Finanzierung überproportional stark und ziehen damit die Produktivität der Branche nach oben. Gemessen an der Wertschöpfung betragen die Produktivitätsgewinne im Durchschnitt aller Branchen zwischen 1,1 und 2,1 \% während fünf Jahren. Die Forscher schätzen, dass dieser Effekt vor allem von der stärkeren Expansion junger, kleiner Firmen getrieben ist, deren Produktion im selben Zeitraum zwischen 3,3 und 6,2\% zunimmt. Zudem berechnen sie, dass die Deregulierung der lokalen Bankenmärkte die Verzerrungen bei der Ressourcenzuteilung innerhalb einer Branche um $24 \%$ reduziert. Rund $85 \%$ dieses Rückgangs ist auf eine bessere Zuteilung von Arbeitnehmern zu Firmen zurückzuführen. Demnach stellen produktive Firmen mehr Arbeitnehmer ein, wogegen die Belegschaft unproduktiver Unternehmen schrumpft.

Die Produktivitätsgewinne innerhalb einer Branche betragen im Durchschnitt 1.1 bis $2.1 \%$. Die Produktion von kleinen, jungen Firmen nimmt dagegen um 3,3 bis $6.2 \%$ $\mathrm{zu}$.

Banken tragen durch die gezielte Finanzierung vielversprechender Unternehmen dazu bei, die Produktivität zu steigern. Der Abbau von Zutrittshürden und Beschränkungen in Bankenmärkten intensiviert den Wettbewerb und führt zu einer effizienteren Kreditvergabe. Davon profitieren vor allem hochproduktive, junge Unternehmen, welche leichteren Zugang zu Krediten erhalten und daher überproportional rasch wachsen können. 
Open Access Dieses Kapitel wird unter der Creative Commons Namensnennung 4.0 International Lizenz (http://creativecommons.org/licenses/by/4.0/deed.de) veröffentlicht, welche die Nutzung, Vervielfältigung, Bearbeitung, Verbreitung und Wiedergabe in jeglichem Medium und Format erlaubt, sofern Sie den/die ursprünglichen Autor(en) und die Quelle ordnungsgemäß nennen, einen Link zur Creative Commons Lizenz beifügen und angeben, ob Änderungen vorgenommen wurden.

Die in diesem Kapitel enthaltenen Bilder und sonstiges Drittmaterial unterliegen ebenfalls der genannten Creative Commons Lizenz, sofern sich aus der Abbildungslegende nichts anderes ergibt. Sofern das betreffende Material nicht unter der genannten Creative Commons Lizenz steht und die betreffende Handlung nicht nach gesetzlichen Vorschriften erlaubt ist, ist für die oben aufgeführten Weiterverwendungen des Materials die Einwilligung des jeweiligen Rechteinhabers einzuholen.

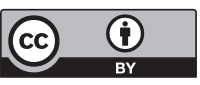

